\section{Pablo Chávez Alayo' Antonio Balarezo Razzeto ${ }^{2}$}

${ }^{1}$ Profesor del Departamento de Clínica Estomatológica de la Universidad Peruana Cayetano Heredia. Lima, Perú.

2Jefe del Programa de Rehabilitación Oral del Postgrado de la Universidad Peruana Cayetano Heredia. Lima, Perú.

\section{Correspondencia}

Pablo Chávez Alayo

Calle Los Nogales Nº164 Urb. Paseo de la Republica-Lima14 Perú

Teléfonos: 511-4676800 / 511-993773695

E-mail: pablo.chavez@upch.pe

\title{
Uso de ponticos ováticos en el sector anterior
}

Chávez-Alayo $\mathrm{P}^{1}$, Balarezo-Razzeto $\mathrm{A}^{2}$. Uso de ponticos ováticos en el sector anterior. Rev Estomatol Herediana. 2012; 22(1):37-41.

RESUMEN

Al realizar un tratamiento en el sector anterior se debe tener una visión amplia, es importante realizar un trabajo multidisciplinario para poder asegurar un resultado óptimo. La pérdida de piezas dentarias en el sector anterior puede traer como consecuencia alteraciones en el reborde edéntulo como la pérdida de las papilas interdentales. Uno de los retos más difíciles al realizar un tratamiento rehabilitador es la preservación de las mismas. Existen diferentes técnicas para poder conseguirlas pero ninguna asegura el éxito a largo plazo. La confección de pónticos ováticos permite preservar las papilas interdentales dando como resultados tratamientos estéticos. A continuación se presenta un caso clínico en el cual se realiza una prótesis fija sobre dos implantes colocados en el sector anterior en el cual se conforman las papilas interdentales con el uso de pónticos ováticos.

Palabras Clave: PÓNTICOS, PÓNTICOS OVÁTICOS, ESTÉTICA EN EL SECTOR ANTERIOR.

Use of ovate pontics in front sector

ABSTRACT

When we treat anterior teeth, we should have a wide vision; it's important to carry out a multi-disciplinary treatment to ensure an optimal result. The loss of anterior teeth can cause alterations in the edentulous ridge like the loss of interdental papillas. One of the most difficult challenges in rehabilitation treatment is the interdental papilla preservation. There are different techniques to get it but none of them ensure a long-term success. The ovate pontic allow the preservation of interdental papilla and also has esthetic results. We present a clinic case of fixed prostheses at two implants in anterior sector with interdental papilla conformation and the use of ovate pontics.

Key Words: PONTIC, OVATE PONTIC, DENTAL ANTERIOR SECTOR.

\section{Introducción}

En la actualidad la odontología no solo se limita a restaurar la estructura dental dañada o ausente, la prevención y reconstrucción de la arquitectura de los tejidos blandos adyacente son unos de los objetivos principales y para eso es necesario realizar tratamientos multidisciplinarios combinando varios procedimientos a fin de alcanzar un resultado altamente estético en la región anterior del maxilar.

La pérdida de piezas dentarias puede ocasionar un defecto óseo en el reborde, y esto representa graves problemas en el clínico, cuando ésta perdida es en el sector anterior, la planificación de la rehabilitación protésica será fundamental para conseguir el éxito deseado. La pérdida de las piezas dentarias en el sector anterior se debe a múltiples factores como trauma, enfermedad periodontal, caries y malformaciones congénitas $(1,2)$.

Uno de los mayores retos para conseguir un tratamiento estético en el sector anterior es la reconstrucción y preservación de las papilas interdentales. La limitada irrigación sanguínea que se encuentra a ese nivel no permite mantener estable la papila interdental (3).

Conseguir la preservación de la papila interdental mediante técnica quirúrgicas es casi imposible, una de las formas es mediante la confección de pónticos ovaticos. El póntico es el elemento suspendido de una prótesis parcial fija que remplaza el diente natural perdido, restaura la función y ocupa el espacio del diente faltante (4).

El motivo por el cual el póntico ovático facilita a la preservación de la papila interdental es porque ésta se encuentra soportada tridimensionalmente, encuentra como base a la cresta alveolar, y la cara proximal de ambos dientes tiene la función de paredes. Al perderse una de las piezas, la papila pierde el soporte y se termina perdiendo pero al traba- jar con el póntico ovático se restablece estas paredes que permitirá la preservación de la papila (5).

Los pónticos son clasificados de muchas maneras, una de las clasificaciones más comunes es según la superficie que se encuentra en contacto con el reborde edéntulo e incluye al póntico esferoide, póntico ovático, póntico de tramo del reborde y póntico del tramo modificado del reborde (6).

Según Kulashekar, existen cuatro tipos básicos de pónticos que han sido usados en los últimos años: el higiénico (sanitarios), en traslape, traslape modificado y el ovático. De los cuatro, el menos estético es el póntico higiénico y está contraindicado utilizarlo en las zonas estéticas. El póntico con traslape total es una buena opción para el sector anterior porque ofrece una buena apariencia estética, el problema es por la forma cóncava de la zona que está en contacto con el reborde edentulo, esto contribu- 
ye a la acumulación de alimentos y facilita la inflamación de los tejidos blandos. Los pónticos con traslape modificado permite una mejor limpieza en comparación que el traslape total, el inconveniente de este póntico es la falta de sellado que no permite hablar al paciente de forma apropiada (7) (Figura 1).

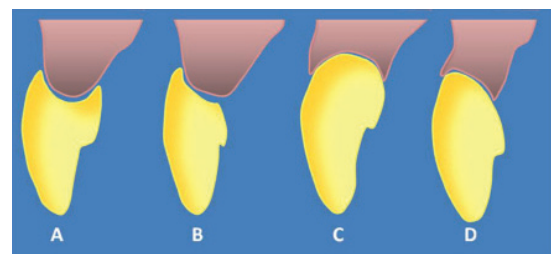

Figura 1. Diseño de los diferentes tipos de pónticos: A. Póntico con traslape total, $B$. Póntico con traslape parcial, C. Póntico ovático, D. Póntico ovático modificado.

El póntico ovático fue desarrollado por Abrams en 1980 (8), en lugar de una superficie cóncava en el tejido superficial, el póntico ovático fue creado con una forma convexa para superar la desventaja de los otros tipos de póntico. Como resultado este póntico es más fácil de limpiar, sin embargo la altura de la superficie convexa fue diseñada cerca del centro de la base y a veces el hilo dental no puede pasar a través del centro del póntico, especialmente en periodontos con un delgado festoneado en los cuales hay una distancia muy larga desde la cima de la papila hasta el margen gingival vestibular. La naturaleza convexa del póntico ovático fue creada para desarrollar un correcto perfil de emergencia, sin embargo en contraste a los requisitos del póntico los cuales sugieren la importancia del contacto sin presión sobre una pequeña área, los pónticos ováticos están en contacto con un área grande de tejido blando inmediato y aplica una muy suave presión.

La ventaja del póntico ovático reside en su capacidad de otorgar máxima estética y en que es usualmente más fácil de limpiar que los pónticos con traslape, asimismo el póntico ovático ofrece un mejor perfil de emergencia y mejores resultados estéticos.

Chiun-Lin $\mathrm{S}$ menciona dentro de varias técnicas para el manejo de reborde que va a recibir una restauración estética la técnica de póntico inmediato que fue descrita por Spear, quien sugirió una forma de mantener la papila interdental seguido a la remoción de piezas anteriores. Se utilizó un provisional modificado para evitar el colapso del alveolo y para imitar el perfil de emergencia natural $(9,10)$.

Existe una modificación del póntico ovático, que implica cambiar la altura del contorno en el tejido de superficie desde el centro de la base a una posición más labial. No requiere demasiado grosor de tejido blando palatino para crear un perfil de emergencia. Es mucho más fácil de limpiar comparado con el póntico ovático debido a su diseño menos convexo. Su mayor ventaja sobre los pónticos ováticos es que usualmente hay una mínima o ninguna necesidad de aumento del reborde mediante cirugía. La altura del contorno en el tejido de superficie del póntico es de 1 a $1.5 \mathrm{~mm}$ apical y palatino al margen gingival bucal. El hilo dental puede ser usado para empujar el margen gingival bucal hacia un lado y limpiar los tejidos de superficie sin ninguna dificultad, en contraste con otros tipos de pónticos. El margen gingival bucal recupera su posición inicial una vez que el hilo dental es retirado. El tejido de superficie del póntico modificado es menos convexo que el del póntico ovático. Está indicado en dientes anteriores y posteriores, con una alta línea de la sonrisa (11).

\section{Reporte de caso}

Paciente de sexo masculino de 54 años de edad, aparentemente sano, y sin alteraciones sistémicas, acude al servicio de postgrado de Rehabilitación Oral de la Facultad de Estomatología de la Universidad Peruana Cayetano Heredia "Roberto Beltrán", cuyo motivo de consulta fue: "mejorar el aspecto de sus dientes de adelante".

Al realizar el examen clínico estomatológico presenta 24 piezas dentarias en boca, 11 piezas superiores y 13 piezas inferiores, espigo muñón colado desadaptado en las piezas 12 y 22, destrucción de las coronas de la piezas 13 y 23 y corona en mal estado de la pieza 14 (Figura 2).

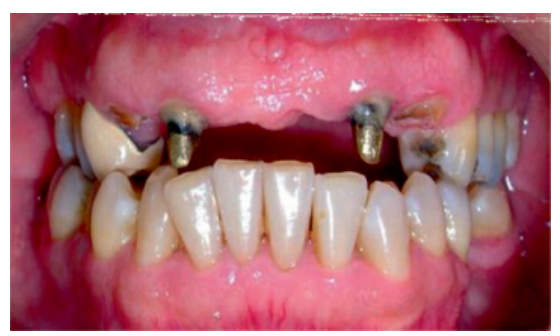

Figura 2. Paciente de 54 años de edad con 24 piezas dentarias en boca. Presenta espigo muñones desadaptados en las piezas 12 y 22, destrucción coronaria en las piezas 13 y 23.

Los exámenes auxiliares que se utilizaron fueron:

1) Modelos de estudios montados en ASA.

2) Encerados diagnóstico.

3) Radiografía panorámica (Fig. 3) 


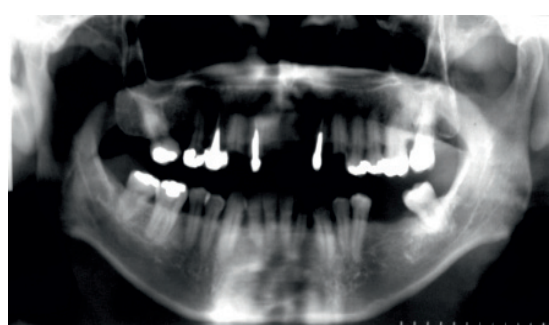

Figura 3. Radiografía panorámica, donde se observa la ausencia de las piezas antero superiores, y las condiciones optimas para colocar los implantes en el sector anterior.

4) Radiografías seriadas.

5) Fotografías intraorales y extraorales.

6) Tomografía axial computarizada (TAC).

7) interconsulta a los servicios de endodoncia, periodoncia e implantología.

\section{Diagnóstico}

Del estado general: paciente de 54 años de edad, de sexo masculino aparentemente sin alteraciones sistémicas.

De las condiciones estomatológicas:

a) Tejidos blandos: Enfermedad gingival inducida a placa dental, deformidades mucogingivales en reborde edéntulo Seibert III en el tercer cuadrante.

b) Tejidos duros: Caries recidivante en las piezas $24,25,26$, 27, 34 y 47. Caries dental en la pieza 38. Atrición en las piezas $33,32,31,41,42$ y 43 . Fractura coronaria en la pieza 15.

c) Diagnostico pulpar: Necrosis pulpar en las piezas 13 y 23, pulpitis irreversible en la pieza 15.

d) Oclusión: Alteración del plano oclusal, interferencia oclusal en movimientos horizontales y verticales, alteración de la guía anterior, edéntulo parcial superior e inferior, Trauma oclusal primario en las piezas 15,45 y 48

y bruxismo céntrico.

Los objetivos del tratamiento fueron: Eliminación de los focos sépticos, educar al paciente en el cuidado y mantenimiento de su salud bucal, educar al paciente en el cuidado y mantenimiento de sus prótesis, mejorar las funciones del sistema estomatognático, mejorar el plano de oclusión, eliminar las interferencias oclusales, mejorar la integridad de las piezas dentarias, restablecer la guía anterior, preservar los tejidos remanentes en boca.

El tratamiento que se propuso al paciente fue: ganancia de corona clínica de las piezas 13 y 23, endodoncia de las piezas 13 y 23, retratamiento de conducto de la pieza 14 , espigo muñón colado de las piezas 14,13 y 23 , colocación de implante post-exodoncia de las piezas $12 \mathrm{y}$ 22, puente metal-porcelana sobre implantes 12-22, coronas metalporcelana de las piezas $13,14,15$, 23 y 26 , puente metal-porcelana sobre las piezas 35-38, incrustaciones de cerómero de la piezas 24,25 y 26 , resina compuesta pieza 27 , resinas en borde incisal para nivel el plano de las piezas $33,32,31,41$, 42,43 y férula oclusal.

Se realizaron las endodoncias de las piezas 13 y 23, se colocaron los implante (Conexao Master Conect Cónico de 4,3 $\mathrm{mm}$. de diámetro y $15 \mathrm{~mm}$. de largo) post exodoncia de las piezas 12 y 22 . Después de tres días de haber colocado los implantes se empiezó a conformar las papilas a nivel de las piezas 11 y 21 con la ayuda de una fresa $\mathrm{N}^{\circ} 3131$ del kit de incrustaciones Inlays de KG Sorensen tomando como referencia de la ubicación de la conformación del talón de los pónticos de las provisionales las cuales fueron previamente confeccionadas (Figuras 4 y 5).

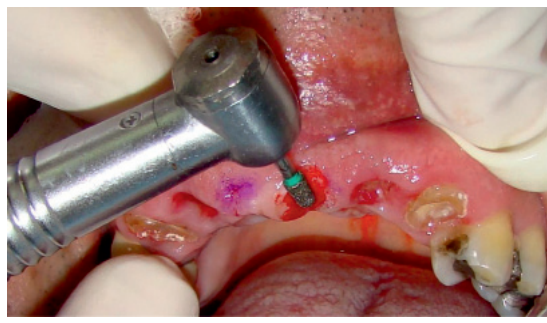

Figura 4. Conformación de la papila interdental con una fresa $N^{\circ} 3131$ del kit de incrustaciones inlay de KG Sorensen, se observa las marcas donde se planifica la conformación de papilas.

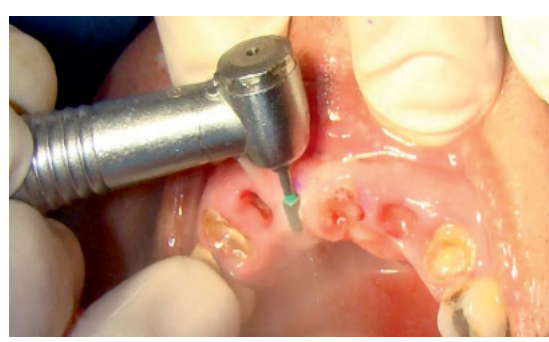

Figura 5. Conformación de papila, es necesario una buena cantidad de tejido.

El talón del póntico de las primeras prótesis provisionales fueron confeccionadas con una forma convexa, uniforme y terminando con un pulido de manera conveniente en todas las superficies, el contacto y la presión sobre la mucosa tuvo que ser mínima. El perfil de emergencia y la longitud del póntico tuvieron que estar en armonía con los pónticos vecinos o dientes pilares, asimismo los contornos linguales confluentes con los dientes vecinos (Figura 6).

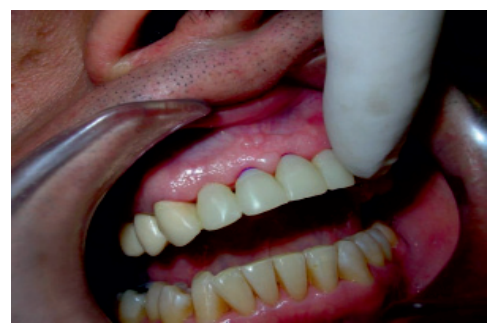

Figura 6. Prueba de la provisional con acrílico termocurado. 
Después de una semana se volvió a confeccionar un nuevo juego de provisionales de acrílico termocurado con refuerzo metálico, se realizaron retenciones en los talones de los pónticos para poder conformar con resina compuesta los pónticos ovativos (Figuras 7, 8, 9, $10,11,12$ y 13$)$.

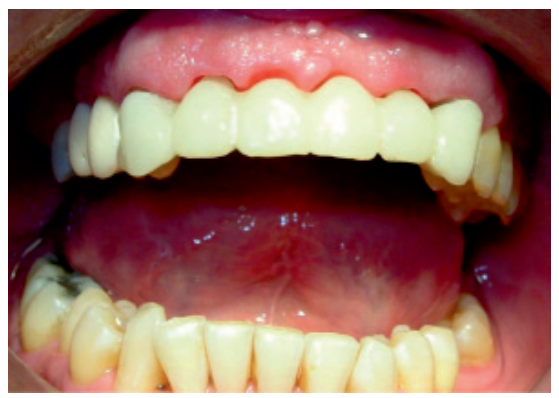

Figura 7. Se observa un espacio entre el talón del póntico y la encía, este espacio será rellenado con resina compuesta.

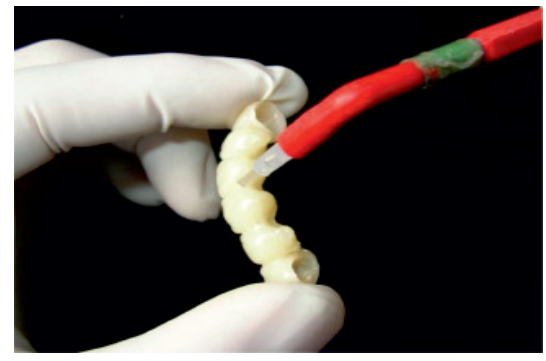

Figura 8.

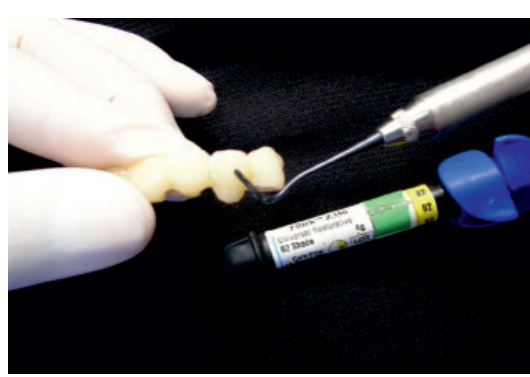

Figura 9.

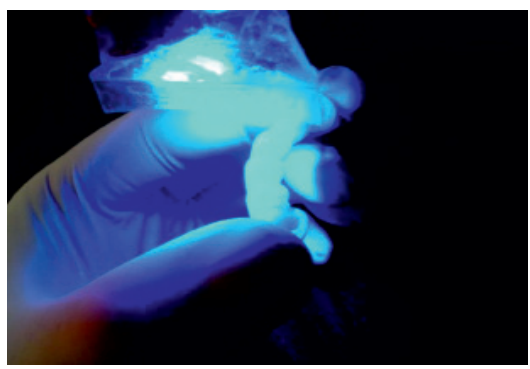

Figura 10.

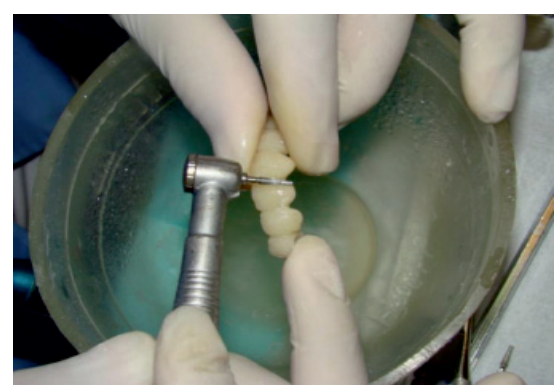

Figura 11.

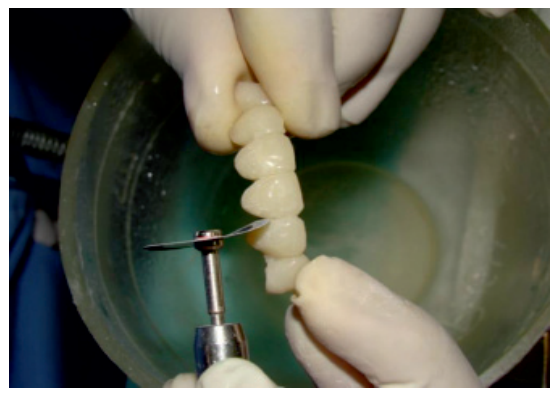

Figura 12.

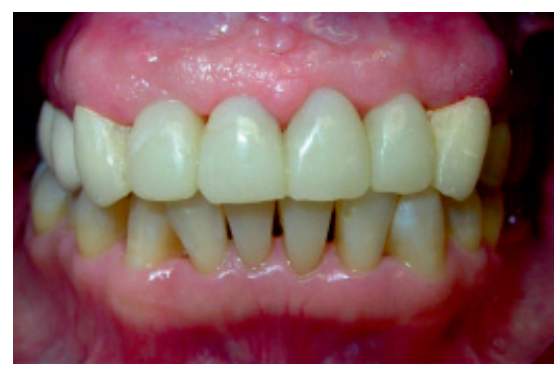

Figura 13.

Figura 7, 8, 9, 10, 11, 12, 13. Confección del póntico ovático con resina compuesta. La resina compuesta asegura una superficie pulida lo que facilitara a la estabilización de los tejidos. La provisional será probado en boca sin ejercer presión sobre los tejidos.

Después de cuatro meses de haber colocado los implantes se realizó la exposición de los mismos, realizando un rebasado de las provisionales. Dos semanas después se inicia la rehabilitación de los implantes a nivel de la pieza $12 \mathrm{y}$ 22. Se realizó una impresión con cubeta abierta con silicona de adición, se ferulizaron los transfers con acrílico duralay (Figuras $14 \mathrm{y}$ 15). Se prueba en boca la estructura metálica de Ni-Cr y se selecciona el color de forma conjunta con las cofias metálicas de las piezas 14,13 y 23 (Figura 16). Luego se probó el bizcocho de las coronas metalporcelana y del puente metal-porcelana.

Las coronas y puente metal-porcelana ya glaseadas son cementadas de manera definitiva con ionómero de vidrio y se realiza la instrucción de higiene oral al paciente con la ayuda de un hilo especial para pacientes portadores de prótesis fija (Figura 17).

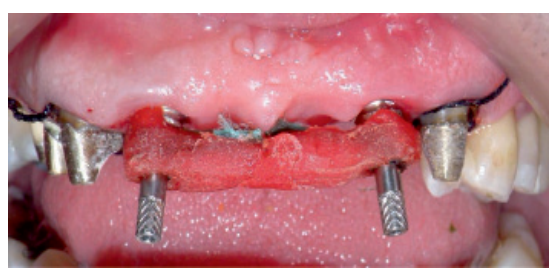

Figura 14. Ferulización de los transfers con acrílico duralay.

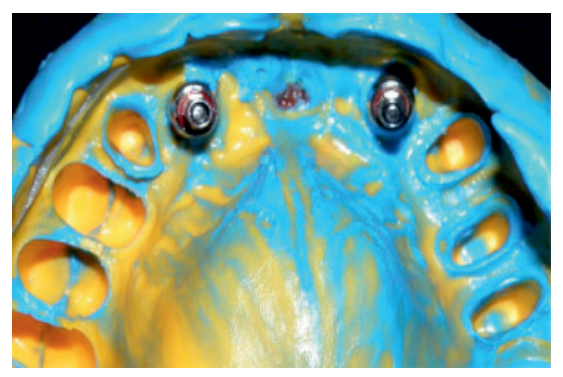

Figura 15. Impresión con silicona por adicción para la confección de la prótesis fija sobre implantes.

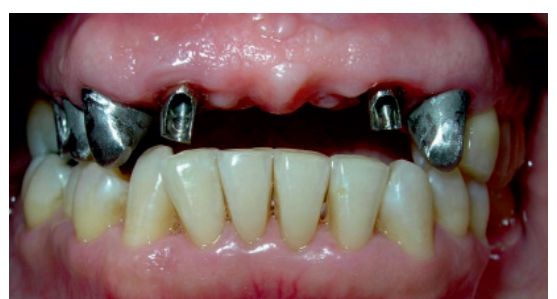

Figura 16. Prueba de los pilares Preparo.

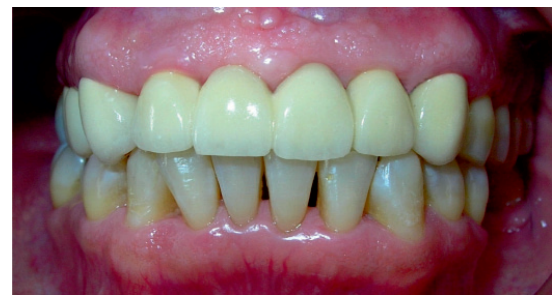

Figura 17. Puente metal cerámica sobre implantes a nivel de las pieza 12 y 22 


\section{Discusión}

Los pónticos ováticos nos permiten conformar una papila en el sector anterior lo cual nos dará buenos resultados en los tratamientos donde existan rebordes edéntulos. La presencia de estos defectos óseos ayudarán a la formación de los triángulos negros los cuales no son aceptados por los pacientes ya que darán una apariencia poco estética.

Kulashekar, menciona cuatro tipos de pónticos que se pueden utilizar: el higiénico (sanitarios), en traslape, traslape modificado y el ovático, cada uno presenta sus ventajas y desventaja, es muy importante saber escoger el póntico de acuerdo a la ubicación que donde se va a utilizar (7).

En el caso clínico presentado, se escogió el póntico ovático porque el paciente presentaba un buen reborde edéntulo con un grosor de encía adecuado, el cual daba la facilidad de conformar de forma satisfactoria la papila; también se pudo escoger el póntico ovático modificado pero al encontrar una buena cantidad de tejido y la demanda estética que exigía el paciente se optó por el primero.

Liu, introduce una modificación del póntico ovático (11), ésta modificación tiene como ventaja facilitar la limpieza por parte del paciente debido a su diseño menos convexo y a la posición más labial del contorno que se encuentra en contacto con el reborde. La posición más labial del este contorno no sacrifica la estética ni mucho menos modifica el perfil de emergencia, por lo cual es una alternativa en rebordes edéntulos con un grosor de tejido deficiente.

En cualquiera de los dos casos: póntico ovático o póntico ovático modificado es necesario realizar un seguimiento al paciente en el cual se controle la oclusión y la limpieza. Asimismo es necesario utilizar varios juegos de prótesis provisionales para asegurar el éxito del tratamiento.

\section{Referencia Bibliograficas}

1. Andrade Acevedo R, Hidalgo de Andrade L, Barbosa e Silva E, Chiérici Marcantonio R. Corrección vertical de reborde edentulo y creación de papila a través de pónticos ovoides. Reporte de caso clínico. Rev Estomatol Herediana. 2010: 20(1):33-37.

2. Zarb JP, Zarb GA. Implant prosthodontic management of anterior partial edentulism: long-term follow-up of a prospective study. J Can Dent Assoc. 2002; 68(2): 92-96

3. Blatz MB, Hurzeler MB, Strub JR. Reconstrution of the lost interproximal papilla presentation of surgical and nonsurgical approaches. Int J Periodontics Restorative Dent. 1999;19:395406.

4. Henriques P. Estética en periodoncia y cirugía plástica periodontal. 1era edición Medellin: Editorial AMOLCA;. 2006.

5. Radz G.M. Soft-tissue considerations in esthetic. reconstruction. Compend Contin Educ Dent. 1998; 19(2):106-11.

6. Tylman S, Malone W. Teoría y práctica de la prostodoncia fija. Medellin: Editorial AMOLCA; 2006.

7. Kulashekar R, Veena H, Aparna I, Dhanasekar B. Incorporating modified ovate pontic design for anterior tooth replacement: A report of two cases. Journal of Indian Prosthodontic Society. 2009; 9 (2):100-104.

8. Abrams L. Augmentation of the deformed residual edentulous ridge for fixed prosthesis. Compend Contin Edu Gen Dent. 1980;1:205-14.

9. Chiun-Lin S. Use of a modified ovate pontic in areas of ridge defects: A report of two cases. J Esthet Restor Dent. 2004; 16: 273-83.

10.Spear FM. Maintenance of the interdental papilla following anterior tooth removal. Pract Periodontics Aesthet Dent. 1999;11:21-28.

11. Liu CL. Use of modified ovate pontic in areas of ridge defect: A report of two cases. J Esthet Restor Dent. 2004;16:273-81. 